\title{
SOME GENERALIZATIONS OF HELLY'S THEOREM ON CONVEX SETS
}

\author{
ALFRED HORN
}

Helly's well known theorem on convex sets states that if $F$ is a family of closed bounded convex sets in $n$-dimensional space such that every $n+1$ members of $F$ have a point in common, then there exists a point which belongs to all members of $F$. The interest of this theorem lies in the role played by the dimensionality of the containing space. If we are given that every $k$ members of $F$ have a point in common, where $k<n+1$, the conclusion is false. In this paper we shall show that significant conclusions can still be drawn in this case.

Consider the case where the sets lie in the plane and every two have a point in common. If we project the members of $F$ on a line $L$, we obtain a family of convex subsets of $L$ to which the hypotheses of Helly's theorem apply. It is an immediate consequence that in any direction there exists a line which intersects all members of $F$. Much less obvious is the statement that through every point there passes a line which intersects all members of $F$. This is the type of result which will be proved in $\$ 4$ below. Instead of parallel projection, we shall project the members of $F$ centrally on a circle (or a sphere, when the dimension of the containing space is greater than two). Then we shall apply, instead of Helly's theorem, a theorem proved below concerning "convex" sets (in the sense described in \$1) lying on a circle (or sphere). Such sets (in a more restricted sense) were considered in a paper by C. V. Robinson [2]. ${ }^{1}$ In that paper the author was interested in conditions on a family of "strictly convex" sets in order that they all have a point in common. Our interest however is in proving a theorem for an arbitrary family of "convex" subsets of a sphere with the only restriction being that every $k$ members of the family have a point in common. This is carried out in $\$ 3$.

I wish to express my thanks to my colleague F. A. Valentine, with whom I had helpful discussions. A special case of Theorem 3 was proved in our joint paper [1].

1. Definitions and notation. We denote $n$-dimensional Euclidean space by $E_{n}$, and if $x=\left(x_{1}, \cdots, x_{n}\right)$ is a point of $E_{n}$, we write

$$
|x|=\left(x_{1}^{2}+\cdots+x_{n}^{2}\right)^{1 / 2} \text {. }
$$

Presented to the Society, November 27, 1948; received by the editors July 8, 1948.

1 Numbers in brackets refer to the references cited at the end of the paper. 
A subspace of $E_{n}$, that is, a hyperplane through the origin, is a subset of $E_{n}$ which is closed under addition and scalar multiplication.

The surface of the unit sphere in $E_{n}$, that is, the set $E_{x}\left(x \in E_{n}\right.$, $|x|=1$ ), will be denoted by $S_{n}$. A great $k$-sphere $S_{k}$ is the intersection of $S_{n}$ with a $k$-dimensional subspace of $E_{n}$. It follows that a great 0 -sphere is the empty set, and a great 1 -sphere is a pair of diametrically opposite points of $S_{n}$. A great 2 -sphere is also called a great circle.

A major arc is a closed arc of a great circle which is greater than a semi-circle (this includes as a special case the whole great circle). A minor arc is a closed arc of a great circle which is smaller than a semi-circle. A semi-circle is neither a major nor a minor arc. If $x$ and $y$ are linearly independent points of $S_{n}$, the minor arc joining $x$ and $y$ is the set

$$
E_{z}(z=\alpha x+\beta y /|\alpha x+\beta y|, \alpha \geqq 0, \beta \geqq 0, \alpha+\beta>0) .
$$

The symbol $[x, y]$ is reserved for the minor arc joining $x$ and $y$.

We shall use the term convex for subsets of $S_{n}$ in a sense which is wider than that usually understood.

Definition 1. A subset $A$ of $S_{n}$ is convex if whenever $x$ and $y$ are linearly independent points of $A$, then either the minor or the major arc joining $x$ and $y$ lies entirely in $A$.

It follows that any set consisting of just two diametrically opposite points is convex, and that any subset of $S_{1}$ is convex.

Definition 2. A subset $A$ of $S_{n}$ is strictly convex if whenever $x$ and $y$ are linearly independent points of $A$, then the minor arc $[x, y]$ lies in $A$.

If $A$ and $B$ are sets, we say that $A$ intersects $B$ if $A \cdot B \neq 0$.

2. Convex sets which are not strictly convex. An immediate consequence of definitions 1 and 2 is the following lemma.

LEMMA 1. If $A$ and $B$ are strictly convex subsets of $S_{n}$, then $A \cdot B$ is strictly convex. If $A$ is a convex subset of $S_{n}$ and $S_{k}$ is a great k-sphere, then $A \cdot S_{k}$ is convex.

LEMma 2. Let $A$ be a closed convex subset of $S_{3}$ which contains a major arc $M$ which is a proper subset of a great circle $S_{2}$, and suppose $A-S_{2} \neq 0$. Then there exists a point $z$ in $A-S_{2}$ such that every great circle through $z$ intersects $A$ in a major arc.

Proof. It will suffice to find an interior point $z$ of $A$ such that $-z \in A$. Let $x$ and $y$ be the end points of $M$. If $b \in A-S_{2}$, all the minor arcs joining $b$ and points interior to $[-x,-y]$ lie in $A$, since 
no point interior to $[x, y]$ is in $A$. Hence $A-S_{2}$ contains an interior point $w$. If $-w$ is not in $A$, all the minor arcs joining $w$ and points of $M$ lie entirely in $A$. Letting $H$ denote the hemisphere bounded by $S_{\mathbf{2}}$ and containing $w$, it follows that $A$ contains $H$ minus the spherical triangle $x, y, w$. Now there exists a point $p$ interior to $[y, w]$ such that $[p, x]$ is not in $A$, for $A$ is closed and $[x, y]$ is not in $A$. Since $A$ is convex, the major arc joining $p$ and $x$ lies in $A$. Clearly, this major arc contains a point $z \in A \cdot H$ with the required properties.

THEOREM 1. If $A$ is a closed convex subset of $S_{n}$ which is not strictly convex, and which is contained in no great $n-1$-sphere, then $A$ contains at least one of every pair of diametrically opposite points of $S_{n}$.

Proof. We proceed by induction on $n$. The cases $n=1$ and $n=2$ are obvious. When $n \geqq 3, A$ contains a major $\operatorname{arc} M$ which is a proper subset of a great circle $S_{2}$. Let $n=3$, and let $z$ be as described in Lemma 2. If $x \in S_{3}-A$, the opposite point $-x$ must lie in $A$ because the great circle through $z$ and $x$ intersects $A$ in a major arc. Now suppose the theorem holds for $n<m, m>3$. Let $z_{1}$ and $z_{2}$ be the end points of $M$. By Lemma 2, if $p \in A-S_{2}$, we can find a point $z$ in the great 3-sphere $S_{3}$ through $S_{2}$ and $p$ such that every great circle on $S_{3}$ through $z$ intersects $A$ in a major arc. Let $z_{3}, \cdots, z_{m}$ be such that $z_{1}, \cdots, z_{m}$ are linearly independent points of $A$, and such that every great circle joining $z_{i}$ to a point of $S_{2}$ intersects $A$ in a major arc, $3 \leqq i \leqq m$. Let $y$ be in the interior of $\left[z_{1}, z_{2}\right]$. Observe $y$ is not in $A$. Now let $x$ be any point of $S_{m}$. We have

$$
x=\alpha_{1} y+\alpha_{2} z_{2}+\cdots+\alpha_{m} z_{m} .
$$

If $\alpha_{3}=0, x$ lies on the great $m-1$-sphere containing $M, z_{4}, \cdots, z_{m}$. By the induction hypothesis, it follows that either $x$ or $-x$ is in $A$. If $\alpha_{3} \neq 0$, we have

$$
x=\alpha_{1} y+\beta w+\alpha_{4} z_{4}+\cdots+\alpha_{m} z_{m},
$$

where $\beta w=\alpha_{2} z_{2}+\alpha_{3} z_{3}, \beta=\left|\alpha_{2} z_{2}+\alpha_{3} z_{3}\right|$. Note that either $w$ or $-w$ is in $A$. Clearly, the points $y, w, z_{4}, \cdots, z_{m}$ are linearly independent. The great $m-1$-sphere through $y, w, z_{4}, \cdots, z_{m}$ intersects $A$ in a closed convex set $B$ by Lemma 1 . Also $B$ contains a major arc which is a proper subset of the great circle through $z_{4}$ and $y$, and $B$ contains $m-1$ linearly independent points $-y, z_{4}, \cdots, z_{m}, \pm w$. Hence by the induction hypothesis, either $x$ or $-x$ is in $B$, and therefore in $A$.

\section{The main theorem.}

TheOREM 2. Let $F$ be a family of closed convex subsets of $S_{n}$ which is 
such that every $k$ members of $F$ have a point in common, $1 \leqq k \leqq n$. Then given any great $n-k$-sphere $S_{n-k}$, there is a great $n-k+1$-sphere containing $S_{n-k}$ and intersecting every member of $F$.

As a noteworthy special case, we have the following theorem.

THEOREM 3. Let $F$ be a family of closed convex subsets of $S_{n}$ which is such that every $n$ members of $F$ have a point in common. Then there exists a point $x$ such that every member of $F$ contains either $x$ or $-x$.

Proof. If we denote the statement of Theorem 2 by $T(n, k)$, then the statement of Theorem 3 is $T(n, n)$. We are going to prove $T(n, k)$ by induction on $n$. The statement $T(1,1)$ is trivially true. The rest of the proof is divided into three parts.

Part 1. If $1 \leqq k \leqq n, T(k, k)$ implies $T(n, k)$.

Proof. Let $E_{n-k}$ be the subspace generated by $S_{n-k}$ and let $E_{k}$ be the subspace of points orthogonal to $E_{n-k}$. We may suppose that no member of $F$ intersects $S_{n-k}$, for otherwise it would intersect every great $n-k+1$-sphere containing $S_{n-k}$. If $x \in E_{n}$, let $P(x)$ denote the projection of $x$ on $E$. By our assumption, if $x \in A \in F$, then $P(x) \neq 0$. Let $A^{\prime}=E_{z}(z=P(x) /|P(x)|, x \in A)$, and $F^{\prime}=E_{B}\left(B=A^{\prime}, A \in F\right)$. It is easily seen that $A^{\prime}$ is a convex subset of $S_{k}=S_{n} \cdot E_{k}$, whenever $A \in F$. In fact, if $z$ and $z^{\prime}$ are linearly independent points of $A^{\prime}$, that is, $z=P(x) /|P(x)|, z^{\prime}=P\left(x^{\prime}\right) /\left|P\left(x^{\prime}\right)\right|$, where $x$ and $x^{\prime}$ are linearly independent points of $A^{\prime}$, then whenever $\alpha^{2}+\alpha^{\prime 2}>0$

$$
\frac{\alpha z+\alpha^{\prime} z^{\prime}}{\left|\alpha z+\alpha^{\prime} z^{\prime}\right|}=\frac{P\left(\beta x+\beta^{\prime} x^{\prime}\right)}{\left|P\left(\beta x+\beta^{\prime} x^{\prime}\right)\right|}=\frac{P\left(\beta x+\beta^{\prime} x^{\prime} /\left|\beta x+\beta^{\prime} x^{\prime}\right|\right)}{\left|P\left(\beta x+\beta^{\prime} x^{\prime} /\left|\beta x+\beta^{\prime} x^{\prime}\right|\right)\right|},
$$

where $\beta=\alpha /|P(x)|, \beta^{\prime}=\alpha^{\prime} /\left|P\left(x^{\prime}\right)\right|$. Hence, whenever $w$ is on the minor (or major) arc joining $z$ and $z^{\prime}$, it is the image of a point on the minor (or major) arc joining $x$ and $x^{\prime}$. Moreover $A^{\prime}$ is closed whenever $A$ is closed. Furthermore every $k$ members of $F^{\prime}$ have a point in common. By $T(k, k)$ there is a point $w$ such that every member of $F^{\prime}$ contains $w$ or $-w$. But the statement $\pm w \in A^{\prime}$ is equivalent to $P(x) /|P(x)|= \pm w$ for some $x \in A$, that is, $P(x)$ is a multiple of $w$. Thus every member $A$ of $F$ contains a point in the subspace $E_{n-k+1}$ generated by $E_{n-k}$ and $w$, and the great $n-k+1$-sphere $S_{n} \cdot E_{n-k+1}$ intersects every member of $F$. To complete the proof of Theorem 2, we need only show:

Part 2. If $n>1, T(n-1, n-1)$ implies $T(n, n)$.

Proof. Let $F$ be a family satisfying the hypotheses of $T(n, n)$, and suppose $T(n, n)$ is false. Then to each $x \in S_{n}$, we can assign a member $A(x)$ of $F$ such that neither $x$ nor $-x$ belongs to $A(x)$. Since $A(x)$ is 
closed, we can find a number $\delta(x)$ such that neither $z$ nor $-z$ belongs to $A(x)$ whenever $|z-x|<\delta(x)$. The sets $E_{z}\left(|z-x|<\delta(x), z \in S_{n}\right.$, $x \in S_{n}$ ) cover $S_{n}$, and we may select a finite covering of $S_{n}$ from these. Let the corresponding members of $F$ be $A\left(x_{1}\right), \cdots, A\left(x_{p}\right)$. Then no point $x$ is such that all of the sets $A\left(x_{i}\right)$ contain either $x$ or $-x$. If we can prove $T(n, n)$ for a finite family $F$, we shall have arrived at a contradiction. Accordingly, we henceforth assume $F$ is finite.

Next we prove that $T(n, n)$ is true if one of the members, say $B$, of $F$ lies entirely on some great $n-1$-sphere $S_{n-1}$. Let $A^{\prime}=A \cdot S_{n-1}$ for each $A$ in $F$, and let $F^{\prime}$ be the family of sets $A^{\prime}, A \in F$. Every $n-1$ members of $F^{\prime}$ have a point in common, since they have a point in common with $B$. Also by Lemma 1 , every member of $F^{\prime}$ is a closed convex subset of $S_{n-1}$. An application of $T(n-1, n-1)$ completes the proof.

Accordingly, we assume that no member of the finite family $F$ lies entirely on a great $n-1$-sphere. By Theorem 1 , we may assume that every member of $F$ is strictly convex.

To prove $T(n, n)$ for a finite family of strictly convex closed sets, we proceed by induction on the number of members of $F . T(n, n)$ being trivially true whenever $F$ has at most $n$ members, assume $T(n, n)$ holds whenever $F$ has fewer than $r$ members, where $r>n$. Before completing the proof of part 2, we need the following result.

Part 3. Suppose $F$ has $r$ members $A_{1}, \cdots, A_{r}$, and suppose there is a point $x$ such that $x \in A_{1} \cdots \cdots A_{s}$, and $-x \in A_{s+1} \cdots A_{s+t}$, where $s>0, t \geqq 0$, and $s+t \leqq r-1$. Then $T(n-1, n-1)$ implies there exists a point $y$ such that either

(a) every member of $F$ contains either $y$ or $-y$, or

(b) $y$ belongs to $s+1$ members of $F$, or

(c) $y$ belongs to $s$ members of $F$ and $-y$ belongs to $t+1$ other members of $F$.

Proof. Every $n-1$ members of the family $\left\{A_{1} \cdot A_{r}, \cdots, A_{r-1} \cdot A_{r}\right\}$ have a point in common. By Lemma 1 , each of these sets is strictly convex. Since $T(n-1, n-1)$ implies $T(n, n-1)$, there exists a great circle $S_{2}$ containing $x$ and $-x$ which intersects all of these sets. Suppose neither $x$ nor $-x$ belongs to $A_{r}$. If $A_{r} \cdot S_{2}$ consists of a pair of diametrically opposite points $y$ and $-y$, then $\pm y \in A_{i} \cdot A_{r} \cdot S_{2} \subset A_{i}$, $i=1, \cdots, r$, and we have (a). Otherwise, $A_{r} \cdot S_{2}$ is a connected subset of $S_{2}$ which is in the interior of one of the semi-circles bounded by $x$ and $-x$. Moreover, $A_{i} \cdot S_{2}$ is connected, $i=1, \cdots, s$, since $A_{i} \cdot S_{2}$ contains a point in $A_{i} \cdot A_{r} \cdot S_{2}$ as well as $x$. Choose a point $x_{i}$ in each of the sets $A_{i} \cdot A_{r} \cdot S_{2}$, and let $y$ be the nearest of the points $x_{1}, \cdots, x_{s}$ to $x$. Then $y$ must belong to $A_{1} \cdots \cdots A_{s} \cdot A_{r}$, since the minor arc joining 
$x_{i}$ and $x$ must contain $y$. This is case (b).

We now return to the proof of part 2. Suppose $F$ has $r$ members. By the induction hypothesis, there is a point $x$ such that $x \in A_{1}$. . . - $A_{s}$, while $-x \in A_{s+1} \cdots \cdots A_{r-1}$, where $1 \leqq s \leqq r-1$. If $T(n, n)$ is false, by part 3 there is a point $y$ common to $s+1$ members of $F$. Applying part 3 again, we can find a point $z$ such that (b) or (c) hold with $s$ replaced by $s+1$ and $t \geqq 0$. If $z$ is not common to $s+2$ members of $F$, by applying part 3 repeatedly we can eventually find a point common to $s+2$ members of $F$. Repeating the process, we finally arrive at a point common to $r-1$ members of $F$. Another application of part 3 completes the proof.

4. Convex sets in the ordinary sense. In this section we use the term convex in the ordinary sense unless otherwise stated. A hyperplane in $E_{n}$ is a translation of a subspace of $E_{n}$. We define a -1dimensional hyperplane as the empty set.

TheOREM 4. Let $F$ be a family of bounded, closed convex sets in $E_{n}$ which is such that every $k$ members of $F$ have a point in common, $1 \leqq k \leqq n+1$. Then through each $n-k$-dimensional hyperplane there passes an $n-k+1$-dimensional hyperplane which inter sects every member of $F$.

Proof. The case $k=n+1$ is Helly's theorem. Suppose $1 \leqq k \leqq n$, and let $H_{n-k}$ be an $n-k$-dimensional hyperplane. By a change of coordinates, we may suppose that $H_{n-k}$ passes through the origin. We may also assume that no member of $F$ intersects $H_{n-k}$. If $A \in F$, let $A^{\prime}$ be the set $E_{y}(y=x /|x|, x \in A)$. Clearly, $A^{\prime}$ is a closed subset of $S_{n}$. If $y_{1}$ and $y_{2}$ are linearly independent points of $A^{\prime}$, that is, $y_{i}$ $=x_{i} /\left|x_{i}\right|, x_{i} \in A, i=1,2$, and if $\alpha_{1} \geqq 0, \alpha_{2} \geqq 0, \alpha_{1}+\alpha_{2}>0$, then

$$
\frac{\alpha_{1} v_{1}+\alpha_{2} y_{2}}{\left|\alpha_{1} y_{1}+\alpha_{2} y_{2}\right|}=\frac{\lambda x_{1}+(1-\lambda) x_{2}}{\left|\lambda x_{1}+(1-\lambda) x_{2}\right|}
$$

where

$$
\lambda=\frac{\alpha_{1} /\left|x_{1}\right|}{\alpha_{1} /\left|x_{1}\right|+\alpha_{2} /\left|x_{2}\right|} \geqq 0 .
$$

Hence $A^{\prime}$ is a strictly convex subset of $S_{n}$ (in the sense of Definition 2 ). Let $F^{\prime}$ be the family of sets $A^{\prime}, A \in F$. $F^{\prime}$ satisfies the hypotheses of Theorem 2, and we conclude that through $S_{n-k}=H_{n-k} \cdot S_{n}$ there passes a great $n-k+1$-sphere $S_{n-k+1}$ intersecting all members of $F^{\prime}$. Let $H_{n-k+1}$ be the subspace generated by $S_{n-k+1}$. The statement $S_{n-k+1} \cdot A^{\prime} \neq 0$ implies there is a point $x$ in $A$ such that $x /|x| \in S_{n-k+1}$ 
and hence $x \in H_{n-k+1}$. Thus $H_{n-k+1}$ intersects every member of $F$. This completes the proof.

As shown in the proof of Theorem 2 (see part 1) the cases $k \leqq n$ of Theorem 4 can be made to depend on the case $k=n$. It does not appear possible to derive the case in $n$ from the Helly case $k=n+1$.

Upon learning of Theorem 4, S. Karlin and L. S. Shapley [3] gave a short proof using Helly's Theorem and the non-existence of a nonvanishing tangential vector field on a sphere of any dimension such that the vectors at antipodal points are equal. Their method does not yield Theorem 2 .

\section{REFERENCES}

1. Alfred Horn and F. A. Valentine, Some properties of $L$ sets in the plane, Duke Math. J. vol. 16 (1949) pp. 131-140.

2. C. V. Robinson, Spherical theorems of Helly type and congruence indices of spherical caps, Amer. J. Math. vol. 64 (1942) pp. 260-272.

3. S. Karlin and L. S. Shapley, Some applications of a theorem on convex functions, Project Rand, publication P-74, April, 1949.

University of California at los Angeles 